\title{
Re-criticism of Tourism Authenticity Based on Popper's Falsificationism
}

\author{
Liangwei Luo
}

College of Tourism, Sichuan Agricultural Uniersity, Chengdu, Sichuan, 611830, China

Keywords: Popper's Falsificationism, Authenticity in Tourism, Re-criticism.

\begin{abstract}
Authenticity in tourism remains a hot topic since the concept of authenticity first introduced into tourism industry. But till now, authenticity in tourism in tourism still remains unclear. This paper uses Popper's falsificationism to analyze and criticize authenticity in tourism. This paper figures out that authenticity in tourism could probably be classified into objective type and subjective type. As result, this paper points out that authenticity in tourism is a scientific recognition problem. It follows the law of 'Hypothesis_-falsification-Re-hypothesis-Re-falsification...... '. In this point, tourism could in no way have a stable objective authenticity.
\end{abstract}

\section{Introduction}

Professor Yang Zhenzhi and Hu Haixia jointly published a paper titled Criticism of Tourism Authenticity on the 12th issue of 2011 of Tourism Tribune, systematically reviewing various theories on tourism authenticity at home and abroad. This paper has constructed a tourism "authenticity" system consisting of objective authenticity, relative authenticity and absolute authenticity, taking a giant step in the research field of tourism authenticity.

\section{Concept of Authenticity and Tourism Authenticity}

Authenticity is rooted from Greek language "authentes”, meaning "authority” or "being made by someone". According to the New English-Chinese Dictionary-Century Edition, authenticity means reliability, validity, and facticity. The concept of authenticity is a chaotic mixture of philosophy, psychology, and the concept of spirit, and is originally from the application in museum. All states of objects are authenticity, and objects change over time and vary under different cultural backgrounds. Therefore, only relative authenticity works, and metaphysically absolute authenticity doesn't work. But the boundary between authenticity and false is blurred in post-modern times.

Boorstin introduced the concept of "authentic" into tourism for the first time in 1964, and hot discussions and analyses were aroused among the circle of western tourism represented by Mac Cannel (1973, 1976), Cohen (1979) et al. The connotation and denotation of the concept of tourism also are constantly evolving, and the concept of tourism is interpreted in more than 200 ways. The interpretations of tourism seem to have verified a word, "There are a thousand Hamlets in a thousand people's eyes". The understanding and interpretation of tourism vary from person to person (Luo Liangwei, 2012). The concept of tourism and that of authenticity are rich in meaning, which objectively determines the complexity and multiplicity of tourism authenticity. Professor Yang Zhenzhi et al. (2011), in the paper of Criticism of Tourism Authenticity, classified tourism authenticity into factual authenticity from cultural perspective, cognitive authenticity from philosophical perspective and absolute authenticity from doxastic authenticity.

\section{Review of Related Researches on Tourism Authenticity}

Since Mc Cannel introduced the concept of "authenticity" into researches on tourist motivation and tour experience in 1973, "authenticity" has become one of the core concepts for theoretical explanation of tourist attraction. Mac Cannel introduced Goffman's foreground and background theory into tourism research, and proposed the theory of staged authenticity, holding a view that tourists' tourism process is actually a process of seeking authenticity, tourists expect to blend 
themselves into the life of sightseeing place and experience local people's life, but what they can touch is only "staged authenticity". Mac Cannel (1976), by borrowing Goffman's foreground and background theory about "staged authenticity", deemed that foreground is shown to tourists, while background is the real scene of sightseeing place. Some researches show that cultures and traditions will lose their original meanings if they are commercialized in tourism (Greenwood 1989; Hughes 1989; Rajotte Tubanavau 1982). The tourist activities and facilities in sightseeing place are presented to tourists in the form of setting, and what tourists see at foreground is merely staged authenticity.

Generally, foreign research results are mainly about objectivism authenticity, constructivism authenticity, postmodernism authenticity, and existentialism authenticity. Objectivists tend to think only experts and anthropologists can accurately grasp the concept of authenticity, and tourists should be excluded from valuators; constructivists recognize that tourists participate in the construction of the concept of authenticity, and authenticity is not objective and changeless; existentialists believe anyone can appraise authenticity, because the authenticity from existential perspective refers to people's subjective experience. Postmodernists, holding an opposite viewpoint, think there is no clear boundary between authenticity and falseness, and there is no concept of authenticity. Besides, they also discriminate tourism type for evaluation criterion.

In 1999, Wang Ning introduced the concept of “authenticity” originated from western scholars' researches on tourism motivation and experience in the early 1970s into the circle of Chinese tourism research. Wang Ning (1999) proposed the existential authenticity of tourism, and considered tourism authenticity has nothing to do with the authenticity of objects at sightseeing place, and tourism authenticity refers to tourists' experience and perception. What Wang emphasized is that tourists confirm and seek their existence via authentic experience.

The researches on tourism authenticity in China are obviously posterior to that overseas, and most of the existing researches are descriptive papers, and there is few theoretical research literature or empirical research literature that is logically rigorous. Few discussions are aroused on authenticity in tourism research. Yu Lan briefly summarized researches on authenticity of cultural tourism overseas, and insisted that this project should focus on experience of authenticity rather than the meaning of authenticity. Professor Xie Yanjun (2006) considered that that "authenticity" is introduced into the field of tourism research before being clearly defined has caused confusion, and researches usually try to figure out the meaning of this term by instinct in many cases.

Yang Zhenzhi and Hu Haixia (2011) classified tourism authenticity into factual authenticity from cultural perspective, cognitive authenticity from philosophical perspective and absolute authenticity from doxastic authenticity, having greatly promoted the process of perceiving tourism authenticity. It is pointed out in the paper that absolute authenticity exists only from doxastic authenticity. This means that absolute authenticity is relative to tourists' subjective sensation only, and tourists' authentic feelings and experience are absolute authenticity of tourism. This paper touches the nature of tourism from philosophical perspective via in-depth analysis with penetrating criticism, but the issues of tourism authenticity still remain complicated and confusing. Then, what are the issues of tourism authenticity about? Is tourism of authenticity? Such questions are still outstanding.

Reviewing previous researches, the writer finds that researches are from different logical perspectives when discussing authenticity issues, including philosophical perspective (such as objective authenticity, existential authenticity, and natural survival authenticity), and cultural fact perspective (staged authenticity and life authenticity). As a result, the issues falling into different logical perspectives are discussed from a perspective, the discussions on authenticity issues are in utter disorder, and the tourism authenticity touched is of an artificially constructed structure without an exception that is of ambiguity and relativity. Thus, it is necessary to further clarify tourism authenticity. The confusion in discussions on tourism authenticity has something to do with the inherent contradictions of its concept. The writer thinks it is of no meaning to discuss tourism authenticity without considering specific tourism phenomenon. 


\section{Re-criticism of Tourism Authenticity Based on Popper's Falsificationism}

\subsection{Essence of Popper's Falsificationism}

As the originator of critical rationalism, Karl Popper (1902-1994) considered in his falsificationism that every kind of scientific theory is no more than some guess or hypothesis that is bound to contain fault, and such fault will come to light one day even though it can escape from empirical test temporarily, and is "retorted" or "falsified" by experience. Science moves forward through an iterative process of coming up with an assumption, discovering a mistake, being denied, and coming up with a new assumption. The growth of scientific knowledge doesn't mean accumulation of observation, but overturning a theory and replacing it with a better or more rational theory".

Rational critical spirit is the essence and marrow of Popper's falsificationism. Popper thought science can be falsified only, and cannot be justified. Popper took the "falsifiability" of experience test rather than the "verifiability" of experience test as the determining criterion of scientific from unscientific statements in his falsificationism, and replaced the "trial and error mechanism" of "problem-assumption-retortion" with the "demonstration mechanism" of "observationsummarization-verification" to come up with a new interpretation of the growth of scientific knowledge. But in Popper's "trial and error mechanism”, verification and falsification interact with each other, move in circles and have dynamic development. In other words, the falsification principle can be used for falsifying universal proposition, and the verification principle also can be used for verifying singular proposition. The falsification principle and the verification principle are complementary to each other. Logically, they have their own advantages that the other doesn't have.

\subsection{Re-criticism of Tourism Authenticity}

In Popper's opinion, science is a kind of cognitive activity, hypothesis and guess are the source of scientific theory, and scientific theory can be falsified only and cannot be verified. Some scientific conjectures can never be falsified, and so have no solutions and are invalid. Similarly, questions like "what tourism authenticity is" are invalid, because it is impossible to infer any objective and eternal truth from general to specific as put in Popper's falsificationism. Therefore, tourism has local authenticity only, and has no absolute and objective authenticity.

Discussers of tourism authenticity all made a prerequisite mistake, assuming that tourism is of invariable authenticity. The ones who preset the objective existence of tourism authenticity first and then explore the authenticity have made a fundamental mistake of metaphysical epistemology. Kant was the terminator of this kind of epistemology. After him, the relativity and finiteness of human rationality were started to be realized. These classic theories unshakable in the past were overturned one by one. The best example is that Newton's classical theory of mechanics was overturned by Einstein's relativity theory. Einstein also admitted that relativity theory is just a transitive theory. Enlightened by Einstein, Popper established the falsificationism theory.

Tourism authenticity can be classified into objective authenticity (tourism object) and subjective authenticity (tourism subject), as shown in Figure 1. Tourism object refers to various tourism resources, including humanistic and cultural tourism resources and natural tourism resources. As to humanistic and cultural resources, authenticity refers to tourism resources in utilization relative to tourism resources as they are; as to natural resources, authenticity refers to the degree of human intervention, the less human intervention is, the higher the natural degree of the resources is, and the naturalness of man-made natural resources. Subjective authenticity is based on tourism theme, namely the authenticity in tourists' subjective perception. Such kind of authenticity varies from person to person, and different subjects with the same objective and the same subject with different objectives have different authenticity perceptions. The spiritual experience of tourism subject of "going deep into the site, and living there cozily" is the highest state of subjective authenticity. To reach this state, personal knowledge reserve, mental culture and physical condition are of great importance. This state is not the authenticity experience that ordinary people can reach, and only few people can make it. Thus, it is not popular, and it is hard to avoid taking a part for the whole and being 
biased if it is discussed as tourism authenticity. Subjective tourism authenticity and objective tourism authenticity overlap with, interact and affect each other, which are in a complicated binary whole relationship.

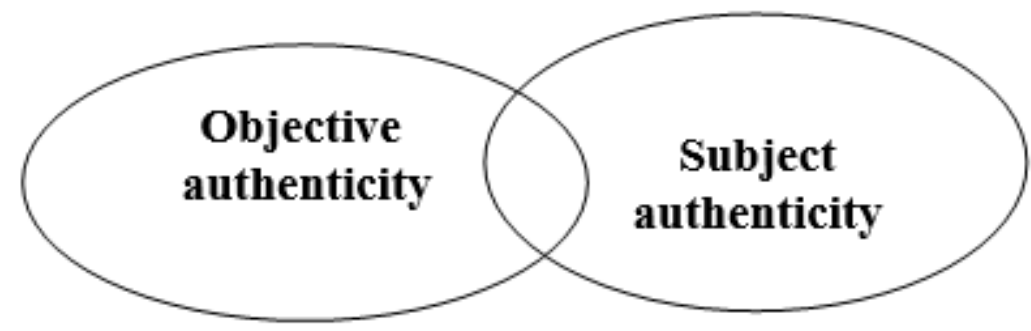

Figure 1. Sketch map of tourism authenticity

The writer also thinks tourism authenticity can be classified into tourism subject authenticity and tourism object authenticity, and various viewpoints and theories on tourism authenticity can be included into subject authenticity for discuss, and then into object authenticity. Subject authenticity refers to authenticity from the perspective of tourism subject perception and experience; and also refers to the authenticity from the perspective of tourism object. Since human society and their culture are constantly evolving and developing, however, their authenticity is only a kind of relative authenticity established by the subject.

As a kind of scientific cognition activity, tourism authenticity is a process of constant cycling and advancing and developing in the twists and turns of "assumption- falsification- assumption-f alsification......”. In some way, tourism authenticity, from the perspective of object or subject, doesn't remain unchanged. Instead, it is constantly advancing, developing, changing and getting rich through falsification with historical development. Therefore, tourism authenticity is of timeliness. Besides, tourism authenticity is also of regionalism. The expectations, objectives and beliefs of tourism vary from region to another, and tourism authenticity varies accordingly. The authenticity of tourism resources and of tourists' experience and perception can be discussed, but it is of no real value if we study tourism authenticity as a whole object, because no specific object can be focused due to the ambiguity and obscurity of the concept. Tourism authenticity is a complicated concept constituted by subjective authenticity and objective authenticity, and a kind of scientific cognitive activity constructed on the basis of tourism phenomena.

\section{Summary}

The recognition and construction of tourism authenticity in a specific historical period in a specific region are theoretical supposition based on certain assumption and guess. According to Popper's falsificationism theory, all theoretical suppositions and assumptions can be falsified and cannot be verified, and falsifiability is the intrinsic quality of scientific theory. The tourism authenticity theory itself is figured out on the basis of assumption and supposition, keeps advancing in the cycle of "assumption-falsifaction-assumption-falsifaction”, and doesn't remain unchanged. The process of endlessly cycling is the intrinsic quality of the tourism authenticity theory. If we take tourism authenticity as an objective entirety, the absolute tourism authenticity is of no meaning. Tourism authenticity is of meaning and value only when referring to specific tourism phenomenon of specific tourism object in a specific period. In a word, tourism authenticity is a general concept. Analysis with Popper's falsificationism theory show that tourism is of no objectively global authenticity. Scholars also can touch local authenticity of tourism phenomenon only, and any construction of tourism authenticity theory with a purpose of surpassing locality is artificial and subjective. Tourism will never be of absolute authenticity. 


\section{References}

[1] He Qiaoli, Wu Weiqiang, Bao Jianqiang. Popper's Falsificationism and the Significance in Scientific Methodology thereof, Social Sciences Review, Issue 1, Volume 11, 2012.

[2] Sun Mingxiang, Chenna. Brief Analysis of Popper's Falsificationism-Popper's Solution to "the Problem of Demarction” and "the Problem of Induction”, Journal of Central South University, 2008 (2).

[3] Wang Ning. Rethinking Authenticity in Tourism Experience, Annals of Tourism Research, 1999, 26(2).

[4] Yang Zhenzhi, Hu Haixia. Criticism of Tourism Authenticity, Tourism Tribune 2011 (12).

[5] Yu Lan. On Researches on Tourism Authenticity, Journal of Beijing Foreign Studies University, 2000 (3). 